\title{
Thyroid Associated Orbitopathy (TAO): Effects of Medical And Surgical Treatments
}

\author{
Dr.Puneet Kumar ${ }^{1},{ }^{*}$ Dr.Naveen Sirohi ${ }^{2}$,Dr.Nitin Tiwari ${ }^{3}$ \\ 1'(M.S, Senior Resident, Surgery, M.L.B. Medical College,Jhansi,Uttar Pradesh,India) \\ ${ }_{2}^{2}$ (M.S, Senior Resident, Ophthalmology, M.L.B. Medical College,Jhansi,Uttar Pradesh,India) \\ ${ }^{3}$ ( Junior Resident, Community Medicine, M.L.B. Medical College,Jhansi,Uttar Pradesh,India' \\ *Corresponding author: Dr.Naveen Sirohi2 *
}

\begin{abstract}
:
Purpose: To assess the effects of treating hyperthyroidism with antithyroid drugs, iodine-131 and surgery on development or aggravation of Graves' ophthalmopathy (TAO) .

Methods: A prospective analysis of 52 patients diagnosed with hyperthyroidism caused by Graves' disease in Department of Surgery and Ophthalmology, Maharani Laxmi Bai Medical College, Jhansi from November 2014 to May 2017 was performed. The patients were stratified in two study groups: groupl(age 20 to 55 years) received treatment with methimazole or iodine-131 and group 2(20 to 55 years) cases underwent subtotal thyroidectomy. The main outcome measures included clinical findings of ophthalmopathy, radiological features (NCCT head with orbits) and treatment options.

Results: 12 patients(23\%) presented with infiltrative ophthalmopathy at randomization while 22 cases $(42.3 \%)$ had palpable thyroid mass. The mean age (years) was $44.8 \pm 4.68$ (range 20-55) and $46.6 \pm 5.53$ (range 2055) in group 1 and 2 respectively. During follow-up period of 12 months, ophthalmopathy developed in 14 cases $(27 \%)$ amd worsened in 10 cases(19.2\%).

Conclusion: In our study both groups showed development or exacerbation of orbitopathy after medical and surgical treatments, however iodine-131 treatment showed more number of cases for the same as compared to methimazole and surgery.
\end{abstract}

Keywords: Graves' Disease, Thyroid Associated Orbitopathy, Subtotal Thyroidectomy, I-131, methimazole

\section{Introduction}

Graves' orbitopath y (GO) is the most common extra-thyroidal manifestation of Graves' disease (GD ), occurring in $25-50 \%$ of patients with the disease ${ }^{[1]}$. GO may occur during or after the onset of hyperthyroidism and less frequently, in euthyroid or hypothyroid patients. The close clinical association between immunogenic hyperthyroidism and orbitopathy suggests that the antigen responsible for these diverse conditions may be shared by the thyroid gland and orbital tissues ${ }^{[2]}$. The incidence of TAO tends to show a bimodal peak, with the first peak between 40 and 50 years of age and the second occurring between 60 and 70 years of age, and occurs in women about 10 times more frequently than in $\operatorname{men}^{[3]}$. The ocular involvement of GD is explained by the expression of

receptor for thyroid-stimulating hormone (TSH-R) present not only in the thyroid follicular cells but also in adipocytes and fibroblasts located in the orbit, and lymphocytes infiltrating orbit tissues ${ }^{[4]}$. Likewise, the signs and symptoms of GO occur due to inflammatory reaction and subsequent fibrosis of the orbital components, including orbital connective tissue, and the extraocular muscles ${ }^{[5]}$. In general, GO can be divided into two clinical stages: the earlier inflammatory (congestive) stage and the late fibrotic (quiescent) stage. The inflammatory stage is marked by edema and deposition of glycosaminoglycans in the extraocular muscles. This results in the clinical manifestations of orbital swelling, proptosis (exophthalmos), diplopia due to restricted ocular motility, periorbital edema, and lid retraction. At this stage the eyes are red and painful. This tends to remit within 3 years and only $10 \%$ of patients develop serious long-term ocular problems. The fibrotic stage is a convalescent phase and may result in further restrictive myopathy and lid retraction. The most serious complication of GO is optic neuropathy, caused by compression of the optic nerve or its blood supply at the orbital apex by the congested and enlarged recti. Such compression, which may occur in the absence of significant proptosis, may lead to severe but preventable visual impairment.

The signs and symptoms of TAO are as varied as the pathophysiology and epidemiology of the disease. Symptoms range from tearing to eye pain to double vision, and signs extend from conjunctival injection and chemosis to lid retraction and exposure keratopathy to strabismus patterns. While approximately half of patients with Graves' disease are asymptomatic from an ophthalmic standpoint, $20-30 \%$ of patients with orbital and ocular involvement are so affected they require targeted therapies ${ }^{[6,7]}$. In principle, thyroid-associated 
ophthalmopathy is a bilateral condition, but it can occur unilaterally in about $20 \%$ of all GO patients ${ }^{[8]}$. Indeed, Graves' disease is the most common cause (20-30\%) of unilateral exophthalmos ${ }^{[9]}$.

The diagnosis of GO is usually made clinically. Among the ocular features, eyelid retraction plays a major role in the clinical diagnosis of the disease. According to Bartley and Gorman's diagnostic criteria ${ }^{[10]}, \mathrm{GO}$ may be diagnosed when eyelid retraction occurs in association with exophthalmos, dysthyroid optic neuropathy(DON) or extraocular muscle involvement. If the diagnosis of thyroid-associated orbitopathy (TAO) can be established clinically, then it is not necessary to routinely order a computed tomography (CT) scan or a magnetic resonance image (MRI). MRI is more sensitive for showing optic nerve compression, whereas CT scanning is performed before bony decompression, because it shows better bony architecture. Neuroimaging usually reveals thick muscles with tendon sparing. The inferior rectus muscle and the medial rectus muscle are usually involved. Bilateral muscle enlargement is the norm; unilateral cases usually represent asymmetric involvement rather than normality of the less involved side. CT findings include: exophthalmos can be measured by drawing a line between the anterior tips of the zygomatic bones and measuring the distance between the line and the posterior part of the eyeball(Fig.1a). The normal distance is more than $10 \pm 1.7 \mathrm{~mm}^{[11]}$ and a smaller distance indicates exopthalmos. Involvement of the extraocular muscles (Fig.1b) in decreasing order of frequency: inferior rectus ,medial rectus, superior rectus, lateral rectus and obliques. Bilateral (76-90\%) and symmetric $(70 \%)$ involvement is typical. The anterior tendon is typically spared (although it can be involved in acute cases), with the swelling largely confined to the muscle belly (Fig.2a), this appearance if often referred to as 'coke bottle' in nature (coca-cola bottle sign) ${ }^{[12]}$. The size of the muscles correlates with both the severity of disease and the risk of optic nerve compression ${ }^{[12]}$. Enlargement of the muscle belly is usually accompanied by reduced attenuation representing fatty infiltration ${ }^{[12]}$. Increase in retro-ocular orbital fat is also seen. The greater the extraocular muscle bulk (especially medial rectus bulk nearer to the apex) and the longer and narrower the bony orbit, the more crowded the orbital apex will become(Fig.2b). Apical crowding could result in optic nerve dysfunction/optic neuropathy due to optic nerve compression ${ }^{[13]}$.

\section{Materials And Methods}

The study was conducted after taking informed consent from all the patients between November 2014 and May 2017, all patients with hyperthyroidism caused by Graves' disease who were referred to us were evaluated for inclusion in the study. All 52 patients who agreed to enter the study were included. The diagnosis of hyperthyroidism caused by Graves' disease was based on the presence of symptoms and signs of hyperthyroidism, a diffuse goiter, elevated total and free thyroxine and total triiodothyronine (T3) concentrations in serum, detectable serum concentrations of thyrotropin receptor antibody (Table1,2). The patients were stratified in two study groups: group1(age 20 to 55 years) received treatment with methimazole or iodine-131 and group 2(20to 55 years) cases underwent subtotal tyroidectomy. The main outcome measures included clinical findings for ophthalmopathy (Table2), radiological features (NCCT head with orbits,Fig.1,2) and treatment options.

Medical therapy consisted of the administration of $10 \mathrm{mg}$ of methimazole four times daily for 12 months. To avert hypothyroidism, thyroxine ( 0.1 to $0.3 \mathrm{mg}$ daily) was added after three to five weeks in a dose that resulted in a normal serum T3 and a low serum thyrotropin concentration. Propranolol (60 to $240 \mathrm{mg}$ per day) or metoprolol (50 to $300 \mathrm{mg}$ per day) was given initially for a few weeks. Methimazole and thyroxine were discontinued simultaneously. The patients were examined monthly for two months after the initiation of treatment and then every three months. After treatment was discontinued, the patients were examined twice during the first year. A single oral dose of iodine-131 was administered to the patients in the iodine subgroup. The dose was 50 milli curie. Unless contraindicated, propranolol or metoprolol was given to all the patients. The patients were examined 6 and 10 weeks after iodine-131 therapy.

The patients who underwent surgery were given propranolol before the operation in a dose of at least $40 \mathrm{mg}$ three to four times daily, or an equivalent dose of metoprolol. The median duration of this therapy before surgery was 10 days. The operation was a bilateral subtotal thyroidectomy, leaving the posterior capsule and approximately 4 to $5 \mathrm{gm}$ of each thyroid lobe ${ }^{[14]}$. After surgery all the patients were given thyroxine to avert hypothyroidism ( 0.1 to $0.3 \mathrm{mg}$ daily). The patients were seen after six weeks, then every three months during the first year after surgery and once yearly thereafter.

\section{Ophthalmologic Follow-up :}

The patients were examined and their ophthalmopathy-index scores (Table3) determined by the ophthalmologist after one year if the first examination had not revealed any signs of ophthalmopathy (i.e., an index score of 0). Patients were referred to the ophthalmologist if eye symptoms or signs developed during follow-up. The examinations included evaluation of soft-tissue changes; measurements of proptosis (by Hertel exophthalmometer), lid width, and lagophthalmos; evaluation of eye-muscle function with the Hess chart; and determination of visual acuity. The activity of ophthalmopathy was scored by the method of Mourits et $\mathrm{al}^{[15]}$., 
which takes into consideration seven manifestations of disease (spontaneous retrobulbar pain, pain with eye movement, eyelid erythema, eyelid edema, conjunctival injection, chemosis, and swelling of the caruncle); one point is given for any manifestation, and the score may range from 0 (no activity) to 7 (very high activity). At each visit, the patients were asked to describe their eye disease as improved, unchanged, or worsened as compared with their last visit. Patients with ophthalmopathy (index score $\geq 1$ ) had monthly ophthalmologic examinations until their condition improved or stabilized; they were then examined one year later. The ophthalmologist was unaware of the type of therapy given except in the cases of patients who underwent surgery and those referred because of ocular problems. We defined the appearance, progression, or improvement of ophthalmopathy according to major and minor criteria. The major criteria were variations of $2 \mathrm{~mm}$ or more in exophthalmometric readings and lid width, the appearance or disappearance of diplopia (intermittent [i.e., present only occasionally], inconstant [i.e., present but not in primary gaze], constant [present in primary gaze]), variations of two points or more in the ophthalmopathy-activity score, and changes of $1 / 10$ or more in visual acuity. Minor criteria were variations in soft tissues or in the patient's assessment of his or her disease. The appearance, progression, or improvement of ophthalmopathy was defined as the appropriate change in at least two major and one minor criteria.

\section{Statistical Analysis}

All the data were stored in Microsoft Excel 2007. All statistical analysis was performed with data analysis software. Descriptive statistics were represented as mean \pm standard deviation. All the tests were twosided and the $\mathrm{P}$ values of less than 0.05 were considered statistically significant. Differences between pretreatment and post-treatment values for exophthalmometric readings, lid-width measurements, and ophthalmopathy-activity scores were compared by the two-tailed Student's t-test; differences in diplopia status (absent vs. present) in the groups were evaluated with the exact binomial test for paired data. Differences among or within treatment groups with respect to the progression of ophthalmopathy and thyroid status were evaluated with the two-tailed Fisher's exact test. Exact binomial 95 percent confidence intervals were calculated for the proportion of patients who had development, worsening, or improvement of ophthalmopathy. There was no financial interests from the authors' side.

\section{Results}

The study had 32 female $(61.5 \%)$ and 20 male (38.5\%) patients . The mean age (years) was $44.8 \pm 4.68$ (range 20-55) and 46.6 \pm 5.53 (range 20-55) in group 1 and 2 respectively. The mean serum T3, thyroxine, and free thyroxine concentrations are shown in Table 1. The serum thyrotropin-receptor antibodies decreased gradually during the first year in the surgically and medically treated subgroups. There were no significant differences in the base-line clinical and biochemical characteristics of the patients in the three treatment groups (Table 1). The proportions of patients with and without ophthalmopathy at base line were similar. 12 patients $(23 \%)$ presented with infiltrative ophthalmopathy (including those with only lid retraction and lid lag) at randomization (Table 3 ) while 22 cases $(42.3 \%$ ) had palpable thyroid mass. During follow-up period (Table 3 )of 12 months, ophthalmopathy developed in 14 cases(27\%) and worsened in 10 cases(19.2\%). In group 1, among the 13 patients $(25 \%)$ treated with radioiodine alone, ophthalmopathy developed or worsened in 10 $(19.2 \%)$ during the year after treatment. In all cases, the changes occurred within the first six months after radioiodine treatment (table 3). The remaining 3 patients treated with I-131 with no ophthalmopathy at base line had no changes in their eyes. While in 13 cases treated with methimazole, 7 patients (13.4\%) developed or worsened ophthalmopathy .The number of smokers was greater among the patients in whom ophthalmopathy developed or worsened than among those who had no change .

In group 2 cases undergoing subtotal thyroidectomy (26 cases), 7(13.4\%) patients had developed or worsened orbitopathy after 12 months follow up. The distribution of the patients with regard to the changes in ocular status is shown in Table 2,3. The ophthalmopathy was mild in the majority (17 cases,70.8\%) of the 24 patients in whom it developed or worsened . The mean maximal index score ranged from 2.4 (group 1, medicaltherapy subgroup) to 3.0 (group 2, medical-therapy subgroup), and the highest score in any patient was 7 . Only 6 of the 24 patients $(25 \%)$, all in group 2, required treatment for ophthalamopathy. No patient lost vision because of optic nerve come pression, and none needed orbital decompression or eyed-muscle surgery. The ophthalmopathy developed or worsened in 10 patients(19.2\%) within the 6 months after therapy began and in 14 patients(27\%) during the rest 6 months of follow up.

\section{Discussion}

Whether treatment of hyperthyroidism in patients with Graves' disease is associated with the development orprogression of ophthalmopathy is the subject of much discussion. We included patients in our study regardless of presence or absence of Graves' ophthalmopathy. During follow up relatively more patients had development of orbitopathy $(18,34.6 \%)$ than worsening of same $(12,23 \%)$. The method of treatment also 
influenced the course of eye changes. The risk of development or worsening was greater (10 out of 13 cases having I-131) in cases treated with I-131 (especially with pretreatment serum T3 level $\geq 5 \mathrm{nmol} /$ litre) than for patients treated with methimazole or surgery. In some retrospective studies, progression of preexisting eye disease was more common than its development after treatment of hyperthyroidism, although progression was not more common after any one of the three treatments - an antithyroid drug, radioiodine or thyroidectomy than after the others ${ }^{[16,17]}$. In some prospective studies ${ }^{[18]}$, radioiodine was associated with progression of ophthalmopathy. Although available comparative studies are limited, at least one prospective trial ${ }^{[19]}$ and two retrospective studies ${ }^{[20,21]}$ indicated that progression of ophthalmopathy was more common in patients treated with radioiodine than in those who received the other two treatments. The development or progression of ophthalmopathy after radioiodine therapy might be related to the release of thyroid antigens as a result of radiation injury and to subsequent enhancement of autoimmune responses directed toward antigens shared by the thyroid and the orbit ${ }^{22]}$. Post treatment hypothyroidism is also suggested in development of ophthalmopathy $^{[23]}$. In our study group 1, patients with high serum T3 levels who took methimazole, risk of development or worsening of ophthalmopathy was comparatively lower ( 7 out of 13 cases taking methimazole) as shown in some studies too ${ }^{[24]}$. In group 2 of surgically treated patients, 7 out of 26 cases undergoing surgery developed or worsened orbitopathy . Some studies had said that ophthalmopathy is not likely to develop or worson after surgery, but this treatment has more risk than methimazole or I-131 $1^{[25]}$.

\section{Figures And Tables}

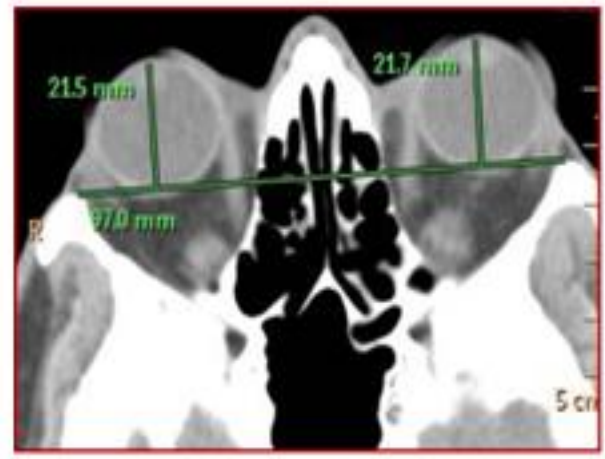

Fig 1a. NCCT head with orbit with transverse view of 47 years old male patients showing bilateral proptosis

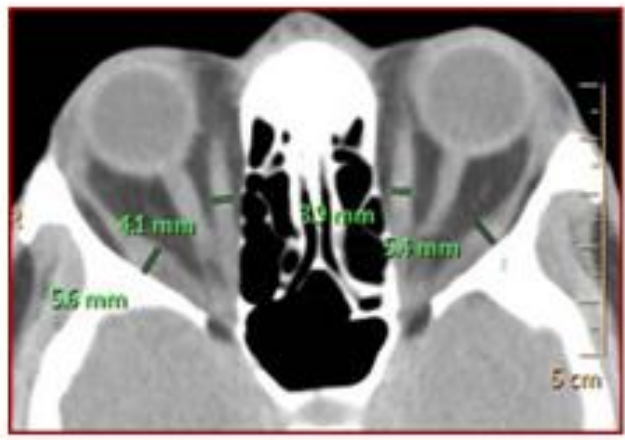

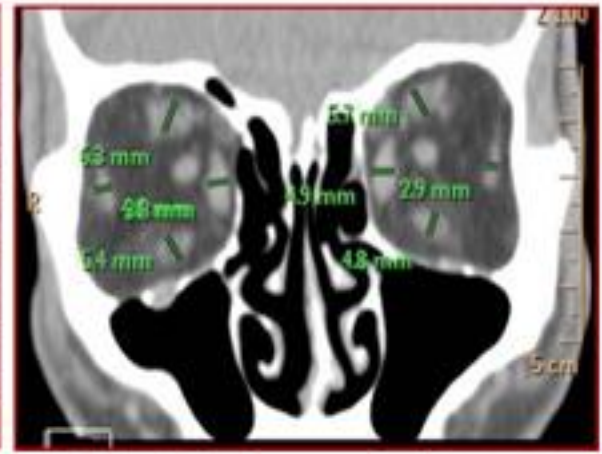

Fig 1b. NCCT head with orbit coronal view of same patient showing bilateral involvement of extra ocular mussels

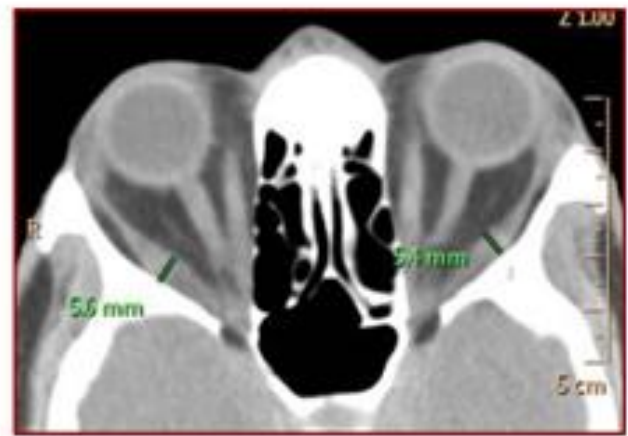

Fig $2 a, 2 b$. NCCT head with transverse view of 29 years old female patient showing enlargement of extraocular muscle belly. 


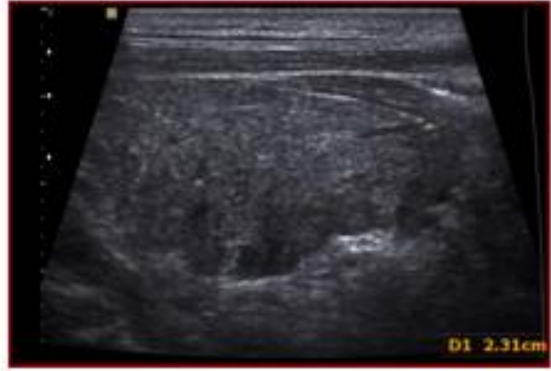

Fig 3a. USG of Thyroid mass in a 42 years female patient showing enlargement of thyroid gland ( $78 \mathrm{ml}$ in volume)

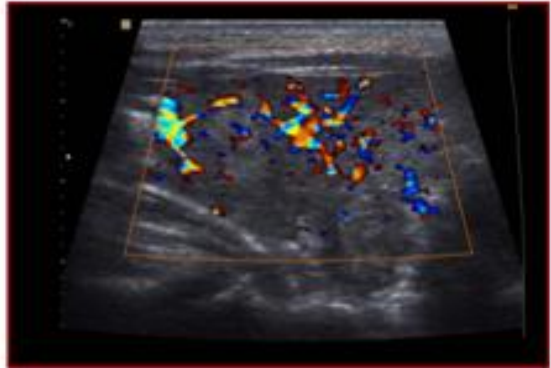

Fig 3b. Colour Doppler of same patient's thyroid gland showing increase blood supply

Table 1: Demographic and laboratory profile of patients $(\mathrm{N}=52)$

\begin{tabular}{|c|c|c|c|}
\hline Mean+SD & $\begin{array}{c}\text { Group } 1 \text { (Methimazole/I- } \\
\text { 131) } \\
\text { n=26 }\end{array}$ & $\begin{array}{c}\text { Group } 2 \text { (Subtotal } \\
\text { Thyroidectomy) } \\
\text { n=26 }\end{array}$ & \\
\hline $\begin{array}{l}\text { Age (In years, mean) } \\
\text { Range 20-55 years }\end{array}$ & $44.8 \pm 4.68$ & $46.6 \pm 5.53$ & \\
\hline $\operatorname{Sex}(M / F)$ & $12 / 18$ & $8 / 14$ & \\
\hline $\begin{array}{l}\text { Duration of } \\
\text { hyperthyroidism } \\
\text { (Range in months) }\end{array}$ & $3-8$ & $5-11$ & \\
\hline $\begin{array}{l}\text { Tobacco smoker } \\
\text { (Number, } \\
\text { Percentage,n=26) }\end{array}$ & $16(62.2 \%)$ & $15(67.7 \%)$ & \\
\hline Labaratory profile & & & Reference range \\
\hline $\begin{array}{l}\text { Mean Thyroid volume } \\
\text { (ml) } \\
\text { (on USG, Fig. 3a ,3b) }\end{array}$ & 44 & 47 & $8-12$ \\
\hline Serum T3 (ng/dl) & $5.8 \pm 1.9$ & $6.1 \pm 2.0$ & $1.1 \pm 2.5$ \\
\hline $\begin{array}{l}\text { Serum total Thyroxine } \\
(\mathrm{nmol} / \mathrm{L})\end{array}$ & $226 \pm 59$ & $237 \pm 67$ & $70-150$ \\
\hline $\begin{array}{l}\text { Serum free Thyroxine } \\
(\mathrm{nmol} / \mathrm{L})\end{array}$ & $57 \pm 17$ & $55 \pm 18$ & $9-21$ \\
\hline $\begin{array}{l}\text { Serum Thyrotropin } \\
\text { Receptor Antibody (U/L) }\end{array}$ & $34 \pm 24$ & $36 \pm 27$ & $<5$ \\
\hline
\end{tabular}

Table 2: Ocular findings of patients before treatment and at 12 months follow up.

\begin{tabular}{|l|l|c|c|c|}
\hline \multirow{2}{*}{ Mean+SD } & \multicolumn{2}{|c|}{ Group 1 } & $\begin{array}{c}\text { Group 2 } \\
\text { (Subtotal } \\
\text { Thyroidectomy) }\end{array}$ \\
\cline { 3 - 5 } & Methimazole & I-131 & \\
\hline Lid width (mm) & Baseline & $11 \pm 1.7$ & $10.7 \pm 2.0$ & $11.2 \pm 1.0$ \\
\cline { 2 - 5 } & Follow up & $13.1 \pm 1.6$ & $12.6 \pm 1.7$ & $12.8 \pm 2.1$ \\
\hline $\begin{array}{l}\text { Proptosis (mm) } \\
\text { (Hertle's } \\
\text { exophthalmometer) }\end{array}$ & Baseline & $17 \pm 2$ & $17 \pm 1$ & $17 \pm 2$ \\
\cline { 2 - 5 } & Follow up & $19 \pm 1.4$ & $18 \pm 2$ & $18 \pm 2$ \\
\hline
\end{tabular}

Table 3: Ophthalmopathy in patients

\begin{tabular}{|c|c|c|c|c|c|}
\hline \multicolumn{2}{|c|}{ Mean+SD } & \multicolumn{2}{|c|}{ Group 1} & \multirow{2}{*}{$\begin{array}{c}\text { Group } 2 \text { (Subtotal } \\
\text { Thyroidectomy) } \\
\text { n=26 }\end{array}$} & \multirow[t]{2}{*}{ Total $(n=52)$} \\
\hline & & Methimazole & I-131 & & \\
\hline \multirow[t]{3}{*}{ Opthalmopathy } & $\begin{array}{l}\text { Present before } \\
\text { study }\end{array}$ & 4 & 3 & 5 & $12(23 \%)$ \\
\hline & Developed & 4 & 2 & 2 & $14(27 \%)$ \\
\hline & Worsoned & 6 & 5 & 3 & $10(19.2 \%)$ \\
\hline \multirow{2}{*}{$\begin{array}{l}\text { Ophthalmopathy } \\
\text { - index score }\end{array}$} & Baseline & \multicolumn{2}{|c|}{$1 \pm 0.6$} & $1 \pm 1$ & \\
\hline & Follow up & \multicolumn{2}{|c|}{$2.4 \pm 0.8$} & $3.0 \pm 1.1$ & \\
\hline \multicolumn{2}{|c|}{$\begin{array}{l}\text { Increase in Exophthalmos (mm) } \\
\text { (Hertle's exophthalmometer) }\end{array}$} & 3.6 & 3.0 & 2.8 & \\
\hline
\end{tabular}




\section{Conclusion}

In our study both groups showed development or exacerbation of orbitopathy after medical and surgical treatments, iodine-131 treatment more likely causing the same as compared to surgery and methimazole. Debates have remained as to how patients with hyperthyroidism due to Graves' disease should be treated. Because antithyroid-drug treatment is not often followed by the development or progression of ophthalmopathy, it might be argued that patients with ophthalmopathy should be treated with such a drug, while waiting for hyperthyroidism and ophthalmopathy to subside spontaneously. However, antithyroid-drug treatment can be associated with unsatisfactory control of hyperthyroidism and, more important, hyperthyroidism can recur after the withdrawal of therapy ${ }^{[26]}$. Persistent hyperthyroidism or its recurrence after the discontinuation of antithyroid-drug therapy could negatively affect the course of ophthalmopathy. Based on our study, we think that it is best to have a permanent cure of hyperthyroidism in patients with ophthalmopathy ${ }^{[27]}$. Subtotal thyroidectomy accomplishes this well. However, the three treatments can be carried out in a multitude of ways and opinions about the treatment of choice do vary ${ }^{[28]}$. Furthermore, a large scale study needs to be undertaken to herald the better and safe mode of treatment for the same.

\section{References}

[1]. Smith TJ. Pathogenesis of Graves' orbitopathy: a 2010 update. J Endocrinol Invest. 2010;33(6):414-21.

[2]. Heufelder AE. Pathogenesis of ophthalmopathy in autoimmune thyroid disease. Rev Endocr Metab Disord. 2000 Jan;1(1-2):87-95.

[3]. Burch HB, Wartofsky L. Graves' ophthalmopathy: current concepts regarding pathogenesis and management. Endocr Rev 1993;14(6):747-93.

[4]. Khoo TK, Bahn RS. Pathogenesis of Graves' ophthalmopathy: the role of autoantibodies. Thyroid. 2007;17(10):1013-8.

[5]. Eckstein AK, Johnson KT, Thanos M, Esser J, Ludgate M. Current insights into the pathogenesis of Graves' orbitopathy. Horm Metab Res. 2009;41(6):456-64.

[6]. Bartalena L. The dilemma of how to manage Graves' hyperthyroidism in patients with associated orbitopathy. J Clin Endocrinol Metab 2011;96(3):592-9.

[7]. Bartalena L, Tanda ML. Clinical practice-Graves’ ophthalmopathy. New Engl J Med 2009;360(10):994-1001.

[8]. Pitz S. Orbital Imaging. In: Wiersinga WM, Kahaly GJ (eds): Graves’ Orbitopathy: A Multidisciplinary Approach. Basel: Karger AG; 2007. p57-65.

[9]. von Arx G. Atypical manifestations. In: Wiersinga WM, Kahaly GJ, eds. Graves' Orbitopathy. A multidisciplinary approach. Basel: Karger AG; 2007. p212-220.

[10]. Bartley GB, Gorman CA. Diagnostic criteria for Graves' ophthalmopathy. Am J Ophthalmol. 1995;119(6):792-5.

[11]. Keats TE, Sistrom C. Atlas of Radiologic Measurement. Mosby. (2001) ISBN:0323001610.pp. 92-95

[12]. Parmar H, Ibrahim M. Extrathyroidal manifestations of thyroid disease: thyroid ophthalmopathy. Neuroimaging Clin. N. Am. 2008;18 (3): 527-36, viii-ix.

[13]. Glatt HJ. Optic nerve dysfunction in thyroid eye disease: a clinician's perspective. Radiology. 1996;200 (1): 26-7.

[14]. Bliss RD, Gauger PG, Delbridge LW. Surgeon's approach to the thyroid gland: surgical anatomy and the importance of technique. World J Surg. 2000 Aug. 24(8):891-7.

[15]. Mourits, M.P, Koornneef, L, Wiersinga, W.M, Prummel, M.F, Berghout, A, van der Gaag, R. Clinical criteria for the assessment of disease activity in Graves' ophthalmopathy (a novel approach) . Br J Ophthalmol. 1989;73:639-644.

[16]. Gwinup G, Elias AN, Ascher MS. Effect on exophthalmos of various methods of treatment of Graves' disease. JAMA 1982;247:2135-2138.

[17]. Fernandez Sanchez JR, Rosell Pradas J, Carazo Martinez O, et al. Graves' ophthalmopathy after subtotal thyroidectomy and radioiodine therapy. Br J Surg 1993;80:1134-1136.

[18]. Kung AWC, Yau CC, Cheng A. The incidence of ophthalmopathy after radioiodine therapy for Graves' disease: prognostic factors and the role of methimazole. J Clin Endocrinol Metab 1994;79:542-546.

[19]. Vestergaard H, Laurberg P. Radioiodine and aggravation of Graves' ophthalmopathy. Lancet 1989;2:47-47.

[20]. Hetzel BS, Mason EK, Wang HK. Studies of serum long-acting thyroid stimulator (LATS) in relation to exophthalmos after therapy for thyrotoxicosis. Australas Ann Med 1968;17:307-311.

[21]. Aron-Rosa D, Perez R, Abitbol Y. Malignant exophthalmos after iodine-131 treatment. Mod Probl Ophthalmol 1973;14:432-434

[22]. Burch HB, Wartofsky L. Graves' ophthalmopathy: current concepts regarding pathogenesis and management. Endocr Rev 1993;14:747-793

[23]. Almqvist S, Algvere P. Hypothyroidism in progressive ophthalmopathy of Graves' disease. Acta Ophthalmol (Copenh) 1972;50:761-70.

[24]. Young ET, Steel NR, Taylor JJ, et al. Prediction of remission after antithy-roid drug treatment in Graves' disease. Q J Med 1988;66:175-89.

[25]. Solomon B, Glinoer D, Lagasse R, Wartofsky L. Current trends in the management of Graves' disease. J Clin Endocrinol Metab 1990;70:1518a 24.

[26]. Vitti P, Rago T, Chiovato L, et al. Clinical features of patients with Graves' disease undergoing remission after antithyroid drug treatment. Thyroid 1997;7:369-375.

[27]. Karlsson FA, Westermark K, Dahlberg PA, Jansson R, Enoksson P. Ophthalmopathy and thyroid stimulation. Lancet 1989;2:691691.

[28]. Glinoer D, Hesch D, Lagasse R, Laurberg P. The management of hyperthya roidism due to Graves' disease in Europe in 1986: results of an international survey. Acta Endocrinol Suppl (Copenh) 1987;285:3-23.

* Dr. Puneet Kumar. "Thyroid Associated Orbitopathy (TAO): Effects of Medical And Surgical Treatments." IOSR Journal of Dental and Medical Sciences (IOSR-JDMS) 16.7 (2017): 33-38. 